\title{
VÍCTIMAS INVISIBLES. ANÁLISIS DE UN CASO DE MALTRATO DESDE LA PERSPECTIVA DE LA VÍCTIMA CON DISCAPACIDAD INTELECTUAL
}

\section{Invisible victims. Analysis of a case of abuse from the perspective of the victim with intellectual disability}

\author{
Belén Gutiérrez-Bermejo \\ UNED. Facultad de Psicología. Departamento de Psicología Evolutiva y de la Educación. \\ C/ Juan Rosal, 10. 28040 Madrid \\ mbgutierrez@psi.uned.es
}

Recepción: 8 de noviembre de 2016

Fecha de aceptación definitiva: 23 de febrero de 2017

RESUMEN: El objetivo de este artículo es analizar la vivencia de maltrato por parte de una persona con discapacidad intelectual. Para ello se utiliza como método de recogida de información una entrevista semiestructurada que fue grabada, transcrita y analizada posteriormente de acuerdo a la literatura científica existente. El resultado de este trabajo pone en evidencia el impacto emocional de la vivencia del maltrato en la víctima, identifica falsos mitos en cuanto a la experiencia emocional de las personas con discapacidad intelectual y aporta explicaciones psicológicas a las actuaciones de las diferentes personas implicadas en las agresiones. La pluralidad de situaciones de maltrato a las que se puede ver sometida la víctima y la permanencia del maltrato a través de los años definen una característica que suele ser habitual en el maltrato hacia las personas con discapacidad intelectual. El entendimiento de toda la dinámica que puede subyacer al maltrato en las personas con discapacidad intelectual posibilita una nueva perspectiva en la evaluación e intervención con este tipo de víctimas.

Palabras Clave: abuso sexual; víctimas; discapacidad intelectual; agresor; evaluación; maltrato; emociones. 
ABSTRACT: This article aims to analyze the experience of abuse by a person with intellectual disability. For this purpose, a semi-structured interview was recorded, transcribed and analyzed according to the existing scientific literature. The results show the emotional impact of the abuse experience on the victim. It also identifies false myths in relation to the emotional experience of people with intellectual disabilities and provides psychological explanations for the actions of the different people involved in the aggressions. The plurality of abuse situations which the victim may face, as well as the permanence of abuse over the years, define a common characteristic in the maltreatment of individuals with intellectual disabilities. Understanding all the dynamics that can lead to abuse in people with intellectual disabilities provides a new perspective in the assessment and intervention with these victims.

KEY WORDs: sexual abuse; victims; intellectual disability; aggressor; assessment; maltreatment; emotions.

\section{Introducción}

Radicionalmente en las investigaciones sobre abuso, son las propias víctimas, ya sean niños o adultos, las que informan sobre sus síntomas y sobre el propio episodio de abuso (Joseph, Williams y Yule, 1997; Yule, 2000). Sin embargo, en el caso de las personas con discapacidad intelectual, habitualmente la información sobre los abusos la proporcionan otras personas (familiares o profesionales) o bien procede de archivos en los que constan casos denunciados. No existen autoinformes sobre maltrato adaptados a las personas con discapacidad intelectual y las entrevistas apenas se realizan por la dificultad que entrañan. Este aspecto puede deberse a los problemas de comunicación de las víctimas o a la ausencia de profesionales formados específicamente en este ámbito con personas con discapacidad intelectual (Callaghan, Murphy y Clare, 2003).

Asimismo, es impensable la utilización de cualquiera de estos procedimientos (autoinforme o entrevista a la víctima) en las personas con discapacidades más graves. En consecuencia, poco se sabe sobre la vivencia de una persona con discapacidad de los abusos sufridos a lo largo de su vida.

A pesar de ello, en esta investigación se ha empleado como método de evaluación la entrevista por las siguientes razones: a) es el método prioritario en la recogida de información de las víctimas de abuso, pues proporciona mucha más información de la que la persona en solitario puede plasmar en un papel (carga emocional, silencios, etc.) (Arruabarrena, De Paúl y Torres, 1996; Cantón y Cortés, 2000); b) supone un primer momento de escucha y de acogimiento para la víctima, y c) las características de esta víctima permitían emplear este método con una adecuada fiabilidad y validez.

Tal y como han confirmado varios autores (Aarons y Powell, 2003, 2006; Bull, 2010; Ternes y Yuille, 2008), las personas con discapacidad intelectual, si son adecuadamente entrevistadas y por profesionales especialistas, pueden proveer testimonios fiables.

Por tanto, el objetivo de esta investigación es analizar el relato obtenido de la entrevista aplicada a una víctima con discapacidad intelectual (Laura). Esta entrevista se 
realizó de acuerdo a las estrategias especiales que requiere su utilización con personas con discapacidad intelectual en casos de abuso (Baladerian, 2004; Nijnatten y Heestermans, 2010). Se trata del caso de Laura.

Laura es una chica de 23 años con un nivel de discapacidad intelectual leve y un porcentaje del 65\% (según el informe emitido por la Gerencia de Servicios Sociales). La puntuación en conducta adaptativa a través del ICAP (Montero, 1996) es 89. Se encuentra laboralmente trabajando en un centro ocupacional.

Laura aceptó voluntariamente contar su caso y que este fuera grabado y utilizado con finalidades científicas. Laura dio a conocer su situación en el curso de formación sobre violencia de género y discapacidad intelectual al que asistió como alumna. Como parte de este curso se grabaron varios testimonios de maltrato. El caso de Laura se eligió entre todos los testimonios para este artículo por las habilidades comunicativas de Laura, su riqueza en cuanto a datos y situaciones, la inclusión de diferentes tipos de maltrato y su capacidad para explicar su vivencia emocional.

Laura cuenta en una sesión de entrevista lo que ha vivido en su infancia. La entrevista fue realizada por una psicóloga experta en abuso en personas con discapacidad intelectual en una sala, con dos sillas dispuestas una al lado de la otra. Se trató de una entrevista semiestructurada, con un breve guion inicial que iba adaptándose a las contestaciones de la persona entrevistada. Se evitó la inducción de cualquier tipo de respuesta fomentando el relato libre. La entrevista se grabó en video y se transcribe a continuación el contenido del relato de la víctima prácticamente de forma literal. Para preservar el anonimato y la identificación de la víctima, los nombres y lugares que aparecen en el relato han sido modificados.

\section{La infancia de Laura}

De pequeña... mi padre pegaba mucho a mi madre, llegaba borracho a casa, fumaba muchísimo, él siempre pedía dinero a mi madre, él nunca tenía dinero. Yo veía cómo mi padre pegaba a mi madre pero no podía hacer nada. Yo era muy pequeña. Luego empezó a pegarme a mí. Me pegaba porque no tenía dinero para fumar y beber. Yo tenía mucho miedo. Al principio me pegaba con la mano, pero un día lo hizo con una rama llena de pinchos y me tuvieron que llevar al hospital para que me sacaran todos los pinchos que tenía clavados. Después murió mi padre. No lo recuerdo muy bien. Estuvo en el hospital y fue cuando mi madre lo abandonó y después me enteré que había muerto. La verdad es que no lo sentí.

Después mi madre se juntó con uno del pueblo, Antonio. A mí me costaba mucho estudiar y como no sabía hacer los deberes del colegio, él me golpeaba la cabeza contra la mesa o contra el fregadero porque decía que no iba a valer para nada. Mi madre no hacía nada porque él le dijo que si quería tener una hija que no valiera para nada. Entonces empezó a pegarme ella también.

Después Antonio dejó a mi madre y mi madre se juntó con otro, Andrés. La vida con él fue parecida, no me pegaba tanto pero me tocaba, me metía mano. 
A los 12 años me bajó la regla y cuando mi madre se enteró, la oí hablar con Andrés diciéndole que yo ya tenía la regla y que ella quería tener un hijo de él y como mi madre no podía tener hijos, pues que lo tuviera conmigo. Andrés siguió metiéndome mano pero no hizo nada más.

Después fue cuando yo tuve un novio unos meses y lo dejamos pero coincidíamos en el colegio. Tenía 12 años, era invierno y yo me levantaba muy pronto para ir al colegio con mi mejor amigo. Un día mi mejor amigo y yo llegamos pronto al colegio y no había nadie en clase, pero de repente oímos pasos. Era mi exnovio y dos amigos, entonces los tres me llevaron a un cuarto que había al lado de la clase y me violaron y nos dijeron a mi amigo y a mí que si contábamos algo nos matarían a nosotros y a nuestras familias.

Yo no hacía más que llorar, pero nadie se daba cuenta. Mi amigo se lo contó a la profesora y ésta reaccionó, estuvo hablando mucho conmigo y se dio cuenta de que también estaba mal alimentada. Me llevaron al médico y vieron que tenía una gran infección genital. Mi madre me dijo que no se me ocurriera contar lo de mi padrastro. Pero intervinieron los servicios sociales y desde entonces estoy en una familia de acogida en la que me quieren mucho. Yo de pequeña pensaba que lo que me pasaba era normal, que eso era lo normal.

Mi nueva madre habló mucho conmigo y me ayudó a superar un poco el miedo a salir con otros chicos.

Ahora tengo 24 años y salgo con un chico. Llevo 9 meses, él me trata muy bien y me cuida mucho.

Recordar todo esto me pone muy triste, me hace sentir lo que sentía cuando era pequeña. Lo que más me dolía no eran los golpes, ni que me tocaran, ni lo que me hicieron en el colegio, sino que sentía que mi madre no me quería.

Ahora veo fotos de personas con moratones y me pongo a llorar, recuerdo cuando mi padre pegaba a mi madre... no puedo ver esas fotos.

\section{Un análisis del relato de Laura}

La vivencia de maltrato de Laura empieza cuando ella era muy pequeña. El maltrato físico ejercido por su padre hacia su madre fue vivido por ella con una gran carga emocional a la que se unió su sentimiento de impotencia para poder impedirlo. Tal y como se deriva de su relato, nada diferencia la vivencia de Laura como testigo de maltrato entre sus padres de la de cualquier otro niño sin discapacidad intelectual. Sentimientos como el miedo y la impotencia cobran una absoluta relevancia (Cantón y Cortés, 2001; Echeburúa, 2004).

La generalización del maltrato por parte del padre a todos los integrantes de la casa es la segunda fase de la victimización vivida por Laura en su infancia, en este caso directamente contra ella. El maltrato hacia ella empieza con golpes y va aumentando hasta el episodio de agredirla brutalmente con una "rama de pinchos", que tiene como consecuencia su ingreso en el hospital. Resulta llamativa la ausencia de sospechas por el personal sanitario del hospital y el silencio de Laura que se instaura como una característica en su forma de actuar. Igual que en cualquier víctima de maltrato que tiene miedo a su agresor o a que los episodios de maltrato sean más graves si revela su victimización o sencillamente a no ser creído (Summit, 1983). 
La ausencia de sentimientos de Laura ante la muerte de su padre no es una manifestación de una falta de emociones en ella sino, más bien, es prueba de su rechazo hacia sus comportamientos con su madre y con ella misma.

Una característica del maltrato en las personas con discapacidad intelectual es su permanencia en el tiempo. La vulnerabilidad al maltrato de las personas con discapacidad no disminuye con el paso del tiempo, a diferencia de lo que ocurre en otras poblaciones vulnerables como los niños prematuros (Verdugo, Bermejo y Fuertes, 1993). Están viviendo durante más tiempo la experiencia traumática que se agrava por su repetición. Algunos autores han puesto de relieve que uno de los factores que agrava la vivencia de las víctimas es que las agresiones se repitan y se produzcan en diferentes momentos del ciclo vital (Echeburúa, 2004; Echeburúa y Guerricaechevarría, 1999). Esta exposición reiterada a la violencia (Reiter, Bryen y Shachar, 2007) daña gravemente a la víctima y le crea un sentimiento de indefensión del cual es muy difícil salir. La indefensión aprendida (Maier y Seligman, 1976; Maier, Seligman y Solomon, 1969; Seligman, 1975; Seligman, Maier y Solomon, 1971) es el estado de resignación pasiva y depresión que surge tras unos fracasos o castigos continuos, sobre todo si estos fracasos y castigos parecen arbitrarios y no dependen de los propios actos. En las personas con discapacidad intelectual a este estado de indefensión se suma la dificultad para entender lo que les está sucediendo (Martinello, 2014). Su limitación intelectual unida a una falta de educación preventiva en el ámbito de la sexualidad, así como la ausencia de estrategias para hacer frente a los hechos que le ocurren, dificulta que elaboren los sucesos traumáticos que les suceden llegando a pensar que "lo que me ocurre es normal”. El maltrato reiterado en la vida de Laura es una constante hasta que intervienen los Servicios Sociales gracias a la participación de su amigo y una profesora.

Tal y como puede apreciarse en el relato de Laura, sucesivos maltratos conformarán su vida posterior sin que nadie haga nada. Esta pasividad, invisibilización o mejor dicho negligencia en la identificación responde a una falta de percepción social de las personas con discapacidad intelectual como víctimas y a unas actitudes negativas hacia ellas (Scior, McLoughilin y Sheridan, 2010; Quamby, 2008, 2011; Deal, 2007; Wilson y Scior, 2015). Esta pasividad es entendida por algunos autores como el resultado de un desequilibrio de poder en la estructuración de los servicios que atienden a las personas con discapacidad. Este desequilibrio de poder es un fenómeno generalizado que impregna la política, los programas que se implantan, la práctica profesional y la dinámica general en las relaciones de las personas con discapacidad (Mandeville y Hanson, 2000). Autores como Ticoll (1994), ya hace varias décadas, alertaron sobre algunas de las formas en que las personas con discapacidad son menos poderosas que quienes abusan de ellas. Adolecen de control sobre las acciones de otras personas que tienen el potencial para invadir o perturbar su vida o el bienestar y, además, carecen de credibilidad o de "voz" si tienen la oportunidad de presentar una queja sobre su mal trato.

El segundo contexto familiar en el que vive Laura nada dista del anterior excepto que en este caso su "nuevo padre" justifica sus acciones violentas contra ella bajo la máscara de una buena acción por su hija (en palabras del primer padrastro: "No quiero que mi hija no valga para nada”). Esta justificación de su acción es asumida 
también por la madre, quién empieza a utilizar los mismos métodos que el padrastro. El padrastro, y por extensión después también la madre, pone en marcha un mecanismo psicológico por el cual se justifica el uso del castigo con un supuesto "fin noble". Hay un cambio de la semántica de la acción y del autor. Esta visión de nobleza en su fin justifica los medios que se están utilizando para conseguirlo (Zimbardo, 2012).

Ante la lectura detallada del relato de Laura, uno se plantea, tal y como numerosos autores han expuesto en el análisis detallado del maltrato hacia las personas con discapacidad intelectual, si en este caso la discapacidad intelectual de Laura no ha podido ser una consecuencia del maltrato físico sufrido en su infancia (Solomons y Young, 1978). Maltrato en forma de repetidos golpes en la cabeza contra el fregadero o la mesa de estudio. Lamentablemente, tal hipótesis nunca podrá confirmarse.

Del maltrato físico en la infancia de Laura se pasa al abuso sexual por su siguiente padrastro (tercera pareja de la madre). En este momento el papel de la madre da un giro y se convierte en instigadora del abuso sexual a su hija, con el fin de que engendre el hijo que ella no puede tener. Todos los límites morales y psicológicos se saltan y Laura es testigo de cómo su madre no solamente encubre los abusos, sino que los justifica y fomenta. El mecanismo psicológico de la disonancia cognitiva (Festinger, 1957) cobra vida en la madre. Cuando se da una discrepancia entre nuestra conducta y nuestras creencias, y cuando los actos no se siguen de unas actitudes pertinentes, se crea un estado de disonancia cognitiva. La persona hace todo lo que pueda para dar alguna forma de coherencia funcional a estas conductas y creencias contradictorias. Cuanto mayor es la discrepancia, más fuerte es la motivación para lograr la consonancia y más extremos son los cambios que se producen. Por extraño que parezca, el efecto de la disonancia es mayor cuanto menor sea la justificación para esas conductas (Festinger, 1957). La madre de Laura, que como tal debía erigirse en adulto protector, permite los abusos sexuales hacia su hija. En esta actuación cobra vida un argumento difícil de digerir por el lector: la posibilidad de que la pareja de la madre conciba un hijo con Laura como si fuera de ella.

Incluso cuando se descubre que Laura tiene una grave infección vaginal, la madre de Laura sigue preocupándose más por su relación de pareja que por su hija, hasta el punto que le dice: “Que no se sepa nada de lo de tu padrastro".

Pero la vida traumática de Laura no acaba ahí, la escalada de violencia llega a su máxima expresión cuando su exnovio participa de su violación en grupo. El mecanismo de deshumanización por parte de los compañeros de clase, entre los que se incluye su exnovio, favorece un acontecimiento tan brutal como este. Laura no era considerada en clase como los demás, era "tonta”; la fuerza de las etiquetas potenció una deshumanización de Laura que permitió un hecho de este calibre (Bandura, Caprara, Barbaranelli, Pastorelli y Regalia, 2001).

La deshumanización es como una catarata en el cerebro que nubla el pensamiento y niega a otras personas su condición de seres humanos. La teoría de la desconexión moral (Bandura, 1991) ilustra la facilidad con la que se puede desconectar la moral mediante la técnica de deshumanizar a la víctima.

A través de la deshumanización, se percibe al otro como despreciable, animal, alguien que no merece vivir. Una de las peores cosas que se puede hacer a otro ser 
humano es privarle de su humanidad, despojarlo de todo valor mediante el proceso psicológico de la deshumanización. Esto sucede cuanto se piensa que los "otros" no tienen los mismos sentimientos, pensamientos, valores y metas que nosotros. Se rebaja o borra de la conciencia toda condición humana que esos "otros" puedan tener en común con nosotros. Se hace mediante los mecanismos psicológicos de intelectualización, negación y aislamiento de las emociones. La deshumanización estigmatiza a los demás y les atribuye una “identidad carente de valor". Erving Goffman (1963) describió el proceso por el que se desacredita socialmente a las personas con discapacidad. No se les considera plenamente humanos y en consecuencia quedan "manchados".

La deshumanización suele facilitar la realización de actos abusivos y destructivos contra las personas que se cosifican de este modo. La deshumanización posibilita incluso culpar a las víctimas de las consecuencias que puedan sufrir (Zimbardo, 2012).

Muchos de los esfuerzos actuales en la concepción de las personas con discapacidad intelectual han incidido en cambiar la forma de denominarles, de modo que esta consideración no dé pie a la deshumanización (Schalok et al., 2010; Verdugo y Schalock, 2010).

Volviendo a Laura, la manifestación que hace de sintomas conductuales tras la violación es evidente: llanto continuo, miedo, depresión, problemas de sueño, ocultación de su victimización. Las personas con discapacidad intelectual, ante sus dificultades comunicativas y de entendimiento de lo que les está ocurriendo en una situación de maltrato y especialmente de abuso sexual, expresan su malestar mediante cambios en su conducta habitual, cambios que muchas veces se catalogan como problemas de comportamiento sin hacerse un análisis riguroso del origen de estos cambios (Lovell, 2007; Peckham, 2007; Taggart, McLaughlin, Quinn y McFarlane, 2007). Cuanto menor es el desarrollo verbal de una persona con discapacidad intelectual más deberán tenerse en cuenta los cambios en su conducta o las manifestaciones conductuales que expresan que algo le está sucediendo (Joseph, Williams y Yule, 1997). Como ya se demostró en una investigación previa que es referente internacional (Verdugo, Bermejo y Fuertes, 1995) y han confirmado estudios posteriores (Brownlie, Jabbar y Beitchman, 2007), dentro de las personas con discapacidad intelectual, las más vulnerables al maltrato son aquellas que tienen dificultades de comunicación.

Las manifestaciones conductuales de Laura ante el suceso sufrido pasan desapercibidas en los entornos en los que ella está inmersa. En palabras de Laura "nadie se daba cuenta", pero ella sí.

En las situaciones en las que se cometen agresiones de este tipo existen los agresores y las víctimas. Pero también suele haber observadores de lo que acontece y que no intervienen para ayudar o para oponerse a esos hechos brutales; con su pasividad permiten que la maldad persista (Zimbardo, 2012).

Tras este análisis de cada uno de los episodios de maltrato vividos por Laura, es el momento de encarar un mito que sigue presente en una parte importante de la sociedad que desconoce el mundo de la discapacidad intelectual, la consideración de que esta población carece de emociones ante hechos traumáticos y que estos hechos no tiene repercusión o impacto en su vida posterior ya que no les afectan, en palabras de la calle, "no se enteran". Esta afirmación es uno de los argumentos que esgrimen los 
agresores sexuales de personas con discapacidad intelectual. Piensan o prefieren creer que sus víctimas no tienen emociones, que el abuso no les afecta, algo que facilita la utilización de los mecanismos de desconexión moral ante la victimización de estas personas.

Consistente con esta afirmación es la actuación profesional que se ha llevado a cabo con las personas con discapacidad intelectual que han sido víctimas de abuso. En muy pocos casos reciben intervención psicológica. Esto es, se castiga al agresor, pero no se trabaja con la víctima. Es una realidad constatada por numerosos autores la inexistencia de tratamiento psicológico para las víctimas con discapacidad intelectual posterior a la identificación de que han sido abusadas sexualmente (Murphy, O’Callaghan y Clare, 2007; Peckham et al., 2007). ¿No es en el fondo esta negligencia otra muestra del mito sobre la falta de impacto emocional de los acontecimientos traumáticos sobre las personas con discapacidad intelectual? $\mathrm{La}$ ausencia de tratamiento a la víctima, como es lógico, sería impensable en las víctimas sin discapacidad intelectual, donde una de las prioridades es trabajar con el que ha sufrido el daño.

Como han evidenciado algunos autores, las personas con discapacidad intelectual, contrariamente a lo que se pensaba ("las personas con discapacidad al tener menos capacidad para comprender sufren menos"), son más vulnerables a la presencia de sintomatología psiquiátrica a partir de un suceso traumático y de situaciones vitales estresantes (Martorell y Tsakanikos, 2008; Murphy, O’Callaghan y Clare, 2007; Robinson y Chenoweth, 2012).

Leyendo el relato de Laura no puede apreciarse ninguna diferencia entre su intensísima vivencia emocional y la de cualquier otra víctima de maltrato y abuso. Es más, tal y como han confirmado algunos autores (Joseph, Williams y Yule, 1997), las víctimas de abuso sexual con discapacidad intelectual pueden presentar la sintomatología del Trastorno por Estrés Postraumático (American Psychiatric Association, 2013). Este trastorno se puede producir cuando una persona ha sufrido un suceso traumático y se compone de cuatro núcleos de síntomas: 1) reexperimentación, 2) evitación conductual/cognitiva, 3) alteraciones cognitivas/estado de ánimo negativo y 4) aumento de la activación y reactividad psicofisiológica.

Si se aplicase la Escala de Gravedad de Síntomas del Trastorno de Estrés Postranmático (Echeburúa, Amor, Sarasua, Zubizarreta, Holgado-Tello y Muñoz, 2016) al caso de Laura, se podría constatar la presencia de múltiples síntomas de este cuadro clínico. Es importante señalar la dificultad que puede entrañar la aplicación de esta prueba a personas con discapacidad intelectual.

Hay que tener en cuenta que el diagnóstico del Trastorno de Estrés Postraumático es más difícil de obtener en personas con discapacidad intelectual por el déficit que les suele acompañar para referir vivencias internas. El Trastorno de Estrés Postraumático en personas con discapacidad intelectual con déficit en la capacidad introspectiva suele manifestarse en forma de trastorno de conducta (McCarthy, 2001).

Puede ser de gran interés una adaptación de esta prueba a personas con discapacidad intelectual; algo que puede posibilitar la identificación de este cuadro clínico en esta población. En definitiva, no es que el hecho de tener discapacidad intelectual 
conlleve ausencia de esta patología, más bien que los instrumentos con los que se cuenta hoy en día no permiten identificar con facilidad esta sintomatología.

Tras leer este testimonio se plantea el hecho de que cómo es posible que Laura haya podido sobrevivir ante tantas situaciones traumáticas; es aquí donde se debe destacar el papel del apoyo social posterior que ha recibido Laura en su familia de acogida.

Tal y como han evidenciado los autores (Echeburúa, 2004), uno de los principales mitigadores del trauma es la existencia de un apoyo social consistente para la víctima.

La victimización secundaria a la que Laura ha estado sometida en los diferentes momentos en los que ha sufrido maltrato se rompe en el momento en el que intervienen su profesora y los Servicios Sociales y, posteriormente, con el cariño y apoyo recibido de su familia de acogida. Existe un acuerdo en la literatura a la hora de afirmar que uno de los principales amortiguadores del impacto del trauma en la víctima es la familia (Cohen y Mandarino, 1996; Malacrea, 2005; Echeburúa y Corral, 2007).

Tal y como se ha comentado anteriormente, los síntomas de estrés postraumático de Laura no han desaparecido, pero ella ahora es capaz de establecer una relación de pareja sana y se siente querida.

Para terminar el análisis del relato de Laura, tal vez uno de los sentimientos más llamativos que expresa en palabras sea la percepción de que su madre "no la quería", un sentimiento que arrastra desde que era pequeña y que pone en evidencia el desarrollo emocional de una persona con discapacidad al margen de su desarrollo cognitivo. Por encima de las conductas de maltrato de la madre hacia ella, se superpone una emoción desgarradora en la víctima que la hace más daño que los golpes sufridos, el sentimiento de falta de amor. Curiosamente ese sentimiento no se da en Laura, que sigue reviviendo el dolor al recordar el maltrato que su padre ejercía contra su madre. En Laura no hay rencor, no hay maldad, sólo prevalece la sensación que ella describe como un nudo en la garganta, como unas ganas horribles de llorar cuando piensa en su madre.

\section{Discusión}

El caso de Laura, en el que se recogen prácticamente todos los tipos de maltrato y donde se ponen en evidencia tanto su permanencia a través del tiempo como la ausencia de respuesta social ante el mismo, no es un caso aislado de maltrato, sino más bien un caso representativo de muchos otros de personas con discapacidad intelectual que permanecen invisibles en la oscuridad.

Existe un gran desconocimiento acerca de la vivencia emocional de las personas con discapacidad intelectual ante situaciones de maltrato $y$, especialmente, en aquellas víctimas de abuso sexual (Robinson y Chenoweth, 2012). Este desconocimiento ha permitido que se generasen falsos mitos sobre el impacto de estas situaciones en ellas, así como que se pasen por alto las señales que pueden manifestar las personas con discapacidad intelectual para expresar que algo les está ocurriendo. Estas manifestaciones, que son habituales en todas las víctimas, tengan o no discapacidad intelectual, cobran especial relevancia en las personas con discapacidad intelectual ante su dificultad para expresar verbalmente lo que les ha ocurrido. 
Hay una gran necesidad de investigación sobre la experiencia emocional de las personas con discapacidad intelectual que permita conocer con más precisión cómo les afectan todas las situaciones que viven a diario.

Por otro lado, es importante poner de manifiesto que la concepción tradicional de las personas con discapacidad -por ejemplo, la consideración de que no sufren o de que sufren menos que las demás personas- ha facilitado la deshumanización y los malos tratos. La abolición de conceptos estigmatizadores, junto con el avance social en el entendimiento de las diferencias, es un campo fértil para dotar de nuevo a las personas con discapacidad intelectual de su "condición humana perdida" por la desconsideración o, mejor dicho, por el desconocimiento de otro ser humano igual que él.

Por otra parte, es fundamental la creación o adaptación de instrumentos de evaluación ya existentes, que permitan detectar el maltrato hacia esta población, explorar el mundo emocional de estas personas y cuantificar la sintomatología que padecen. Esto es especialmente necesario, difícil y sugerente para aquellas personas que tienen mayores dificultades de comunicación. En suma, es una necesidad urgente para poder detectar, identificar y tratar el maltrato y la sintomatología específica en cada persona que tiene discapacidad intelectual.

A su vez, siempre que el nivel de comunicación de la persona lo permita, la entrevista semiestructurada resulta una técnica de evaluación útil y adecuada en la atención y recogida de información de personas con discapacidad intelectual víctimas de malos tratos.

Algo tan básico como considerar de nuevo a la persona con discapacidad como ser humano que siente y está lleno de emociones, así como especialmente vulnerable al maltrato, potenciaría el desarrollo de acciones sociales tan relevantes como: la protección ante los malos tratos, la inversión en investigación y en recursos destinados a la prevención y tratamiento psicológico frente al impacto que puedan producir en ella los acontecimientos traumáticos, que por las condiciones que rodean a su discapacidad intelectual es más fácil que le puedan ocurrir.

\section{Referencias bibliográficas}

Aarons, N. y Powell, M. (2003). Issues related to the interviewer's ability to elicit reports from children with an intellectual disability: A review. Current issues in Criminal Justice, 14, 257-268.

Aarons, N., Powell, M. y Snow, P. (2006). An examination of the questioning styles of police, officers and caregivers when interviewing children with an intellectual disability. Legal and Criminological Psychology, 11, 35-53.

American Psychiatric Association (2013). DSM-5. Diagnostic and statistical manual of mental disorders ( 5 th ed.). Arlington, VA: Author.

Arruabarrena, I., De Paúl, J. y Torres, B. (1996). El maltrato infantil: detección, notificación, investigación y evaluación. Madrid: Ministerio de Asuntos Sociales (2. edición).

BALADERIAN, N. J. (2004). Interviewing skills to use with abuse victims who bave intellectual Disabilities. Washington D. C.: National Aging Resource Center on Elder Abuse. 
Bandura, A. (1991). Social cognitive theory of moral thought and action. En W. M. KurTines y J. L. GewirTz (Eds.), Handbook of moral behavior and development (pp. 45-103). Hillsdale, NJ: Erlbaum.

Bandura, A., Caprara, G. V., Barbaranelli, C., Pastorelli, C. y Regalia, C. (2001). Sociocognitive self-regulatory mechanisms governing transgressive behaviour. Journal of Personality and Social Psychology, 80, 125-135.

Bromley, J. y Emerson, E. (1995). Beliefs and emotional reactions of care staff working with people with challenging behavior. Journal of Intellectual Disability Research, 39 (4), 341-352.

Bull, R. (2010). The investigative interviewing of children and other vulnerable witnesses: Psychological research and working/professional practice. Legal and Criminological Psychology, 15, 5-23.

Callaghan, A. C., Murphy, G. H. y Clare, I. (2003). The impact of abuse on men and women with severe learning disabilities and their families. British Journal of Learning Disabilities, $31(4), 175-180$.

CANTÓN, J. y CORTÉs, M. R. (2000). Guía para una entrevista de investigación. En J. CANTón y M. R. CORTÉs, Guía para la evaluación del abuso sexual infantil. Madrid: Pirámide.

CANTÓN, J. y CORTÉs, M. R. (2001). Sintomatología, evaluación y tratamiento del abuso sexual infantil. En V. E. Caballo y M. A. Simón (Eds.), Manual de Psicología clínica infantil y adolescente. Madrid: Pirámide.

Cohen, J. A. y Mannarino, A. P. (1998). Intervention for sexually abused children: initial treatment outcome findings. Child Maltreatment, 3, 17-26.

Deal, M. (2007). Aversive disablism: subtle prejudice toward disabled people. Disability and Society, 22 (1), 93-107.

Echeburúa, E. (2004). Superar un trauma. Madrid: Pirámide.

Echeburúa, E., Amor, P. J., Sarasua, B., Zubizarreta, I., Holgado-Tello, F. y Muñoz, J. M. (2016). Escala de Gravedad de Síntomas Revisada (EGS-R) del Trastorno de Estrés Postraumático según el DSM-5: propiedades psicométricas. Terapia Psicológica, 3 (2), 111-128.

Echeburúa, E. y Corral, P. (2007). Intervención en crisis en víctimas de sucesos traumáticos: ¿cuándo, cómo y para qué? Psicología Conductual, 15, 373-387.

Echeburúa, E. y Guerricaechevarría, C. (1999). Abuso sexual en la infancia: concepto, factores de riesgo, y efectos psicopatológicos. En J. SANMARTín (Ed.), Violencia contra niños. Barcelona: Ariel.

Festinger, L. (1957). A theory of cognitive dissonance. California: Stanford University Press.

Goffman, E. (1963). Stigma: Notes on the management of spoiled identify. New Jersey: Prentice-Hall.

Joseph, S., Williams, R. y Yule, W. (1997). Understanding Post Traumatic Stress: A Psychosocial Perspective on PTSD and Treatment. Chichester: John Wiley y Sons.

Lovell, A. (2007). Learning disability against itself: the self-injury/self-harm conundrum. British Journal of Learning Disabilities, 36 (2), 109-121.

Maier, S. F. y Seligman, M. E. (1976). Learned helplessness: Theory and evidence. Journal of Experimental Psychology, 105, 3-46.

Maier, S. F., Seligman, M. E. y Solomon, R. L. (1969). Pavlovian fear conditioning and learned helplessness. En B. A. Campbell y R. M. Church (Eds.), Punishment. New York: Appleton.

Malacrea, M. (2000, 2005). Trauma y reparación. Barcelona: Paidós.

Mandeville, H. y Hanson, M. (2000). Understanding caregiver abuse as domestic violence: systemic change in Wisconsin. Impact, 13 (3), 14-15, 27.

Martinello, E. (2014). Reviewing Strategies for Risk Reduction of Sexual Abuse of Children with Intellectual Disabilities: A Focus on Early Intervention. Sexuality and Disability, 32, 167-174. 
Martorell, A. y Tsakanikos, E. (2008). Traumatic experiences and life events in people with intellectual disability. Current Opinion in Psychiatry, 21 (5) 2, 445-448.

McCarthy, J. (2001). Post-traumatic stress disorder in people with learning disability. Advances in Psychiatric Treatment, 7, 163-169.

Montero, D. (1996). Evaluación de la conducta adaptativa en personas con discapacidades. Adaptación y validación del ICAP. 3. a ed. España: Mensajero.

Murphy, G. H., O’Callaghan, A. C. y Clare, I. (2007). The impact of alleged abuse on behaviour in adults with severe intellectual disabilities. Journal on Intellectual Disability Research, 51, 741-749.

Nijnatten, C. Van y Heestermans, M. (2010). Interviewing victims of sexual abuse with an intellectual disability: A dutch single case study. Journal of Social Work Practice, 24 (4), 391-407.

Pecknam, N. (2007). The Vulnerability and sexual abuse of people with learning disabilities. British Journal of Learning Disabilities, 35 (2), 131-137.

Peckham, N., Corbett, A., Howlett, S., McKee, A. y Pattison, A. (2007). The delivery of a survivors' group for learning disabled women with significant learning disabilities who have been sexually abused. British Journal of Learning Disabilities, 35 (4), 236-244.

Quamby, K. (2008). Getting away with murder: Disabled people's experiences of hate crime in the UK. Scope.

Quamby, K. (2011). Scapegoat: Why we are falling disabled person. London: Portobello Books.

Reiter, S., Bryen, D. y Shachar, I. (2007). Adolescents with intellectual disabilities as victims of abuse. Journal on Intellectual Disability Research, 11 (4), 371-387.

Robinson, S. y CHenoweth, L. (2012). Understanding emotional and psychological harm of people with intellectual disability: an evolving framework. The Journal of Adult Protection, 14 (3), 110-121.

Schalock, R. L., Borthwick-Duffy, S. A., Bradley, V., Buntix, W. H. E., Coulter, M. D., Craig, E. M., Gómez, S. C., Lachapelle, Y., Luckasson, R., Reeve, A., Shogren, K. A., Snell, M. E., Spreat, S., Tassé, M. J., Thompson, J. R., Verdugo, M. Á., Wehmeyer, M. L. y Yeager, M. H. (2010). Intellectual disability. Definition, Classification, and Systems of Supports. 11th Edition. Washington, D.C.: American Association on Intellectual and Developmental Disabilities, 2010. [Traducción al español: Verdugo, M. Á. (2011). AAIDD. Discapacidad intelectual. Definición, clasificación y sistemas de apoyo. 11. a ed. Madrid: Alianza Editorial].

Scior, K., McLoughilin, A. y Sheridan, J. (2010). Public attitudes toward people with intellectual disabilities: A cross-cultural study. Intellectual and Developmental Disabilities, 48 (4), 278-289.

Seligman, M. E. P. (1975). Helplessness: On depression, development and death. San Francisco: Freeman.

Seligman, M. E. P., Maier, S. F. y Solomon, R. L. (1971). Unpredictable and uncontrollable aversive events. En F. R. Brush (Ed.), Aversive conditioning and learning. New York: Academic Press.

Solomons, G. y Young, H. A. (1978). Malpractice and child abuse. Journal of the Iowa Medical Society, 7, 239-243.

Summit, R. C. (1983). The child sexual abuse accommodation syndrome. Child Abuse $\&$ Neglect, 7, 177-193.

Taggart, L., Mclaughlin, D., Quinn, B. y McFarlane, C. (2007). Listening to people with intellectual disabilities who misuse alcohol and drugs. Health ESocial Care in the Community, 15 (4), 360-368.

(c) Ediciones Universidad de Salamanca / CC BY-NC-ND

Siglo Cero, vol. 48 (1), n. ${ }^{\circ}$ 261, 2017, enero-marzo, pp. 9-21 
Ternes, M. y Yuille, J. C. (2008). Eyewitness memory and eyewitness identification performance in adults with intellectual disabilities. Journal of Applied Research in Intellectual Disabilities, 21, 519-531.

Ticoll, M. (1994). Violence and People with Disabilities: A Review of the Literature. Canada, Toronto: The Roeher Institute.

Van Nijnatten, C. y Heestermans, M. (2010). Interviewing victims of sexual abuse with an intellectual disability: A Dutch single case study. Journal of Social Work Practice, 24 (4), 391-407.

Verdugo, M. Á., Bermejo, B. G. y Fuertes, J. (1993). Maltrato infantil y minusvalia. Madrid: Ministerio de Asuntos Sociales.

Verdugo, M. Á., Bermejo, B. G. y Fuertes, J. (1995). The maltreatment of intellectually handicapped children and adolescents. Child Abuse E Neglect, 19 (2), 205-215.

Verdugo, M. Á. y Schalock, R. L. (2010). Últimos avances en el enfoque y concepción de las personas con discapacidad intelectual. Siglo Cero, vol. 41 (4), 236, 7-21.

Wilson, M. C. y ScIor, K. (2015). Implicit attitudes towards people with intellectual disabilities: Their relationship with explicit attitudes, social distance, emotions and contact. Plos ONE, 10 (9).

Yule, W. (2000). Post-Traumatic Stress Disorders: Concepts and Therapy. New York: Wiley. Zimbardo, P. (2012). El efecto Lucifer. El porqué de la maldad. Barcelona: Paidós. 